\title{
Equivalent Modelling of Hybrid RES Plant for Power System Transient Stability Studies
}

DOI:

10.1109/TPWRS.2021.3104625

\section{Document Version}

Accepted author manuscript

Link to publication record in Manchester Research Explorer

\section{Citation for published version (APA):}

Radovanovic, A., \& Milanovic, J. V. (2021). Equivalent Modelling of Hybrid RES Plant for Power System Transient Stability Studies. IEEE Transactions on Power Systems. https://doi.org/10.1109/TPWRS.2021.3104625

\section{Published in:}

IEEE Transactions on Power Systems

\section{Citing this paper}

Please note that where the full-text provided on Manchester Research Explorer is the Author Accepted Manuscript or Proof version this may differ from the final Published version. If citing, it is advised that you check and use the publisher's definitive version.

\section{General rights}

Copyright and moral rights for the publications made accessible in the Research Explorer are retained by the authors and/or other copyright owners and it is a condition of accessing publications that users recognise and abide by the legal requirements associated with these rights.

\section{Takedown policy}

If you believe that this document breaches copyright please refer to the University of Manchester's Takedown Procedures [http://man.ac.uk/04Y6Bo] or contact uml.scholarlycommunications@manchester.ac.uk providing relevant details, so we can investigate your claim.

\section{OPEN ACCESS}




\title{
Equivalent Modelling of Hybrid RES Plant for Power System Transient Stability Studies
}

\author{
Ana Radovanović, Student Member, IEEE, Jovica V. Milanović, Fellow, IEEE
}

\begin{abstract}
The paper proposes methodology for de velopment of dynamic equivalent model of hybrid renewable energy source (HRES) plant suitable for reliable assessment of the overall transient stability of the realis tic, large power systems. Transie nt stability index is used for system stability assessment, and consequently for the evaluation of the model performance. The historical plant production data and transmission network shortcircuit fault statistics, together with probabilistically modelled uncertainties associated with system operation, are used to gene rate a set of realis tic dynamic responses of the plant over the period of one year. The use of clustering algorithms enabled unsupervise d grouping/clustering of responses and de ve lopment of the low order equivalent models in the form of a transfer function with voltage as an input and real and reactive power as outputs. The methodology is illustrated on a number of case studies using two HRES plant configurations. It is demonstrated that a very few low order dynamic models can be used to model the HRES plant during the year for sufficiently accurate transient stability assessment of the power system. The possibility of deriving these equivalent models based on forecasted HRES plant composition makes them attractive for practical application in power system dynamic security studies.
\end{abstract}

Index Terms--Data clustering, equivalent dynamic models, hybrid renewable energy plants, transient stability

\section{INTRODUCTION}

$\mathrm{T}$ HE growth in the installation capacity of distributed generation (DG), as well as the trend of displacement of fossil-fueled and nuclear power plants have changed electric power system structure and operation substantially. The new sources are connected to distribution network voltage levels and characterized by stochastic production and power electronics, which makes them considerably different from conventional synchronous generators (SGs) [1]. In order to assess to what extent DG can influence the overall system stability, it is necessary to conduct numerous large system dynamic studies. In the past, DG was traditionally represented as negative static load in network simulations [2]. Nowadays, considerable DG installation capacity imposes more demanding modelling requirements and dynamic models have to be used instead. However, large number of distributed generators makes their detailed dynamic representation impractical as it results in significant computational time for

This work was supported by the EU Horizon 2020 Project CROSSBOW, Grant Agreement 773430.

The authors are with the Department of Electrical and Electronic Engineering, The University of Manchester, Manchester M13 9PL, U.K. (email: ana.radovanovic@manchester.ac.uk; milanovic@manchester.ac.uk). carrying out large system analyses [2, 3]. Furthermore, fullscale dynamic models cannot always be developed due to lack of data and confidentiality issues which prevent the exchange of detailed network models between different network operators $[4,5]$.

Dynamic equivalent models (DEMs), i.e., simplified dynamic representations, have been recommended for overcoming issues related to dynamic modelling of DG plants. Over the years, a number of DEMs of DG plants, as well as DEMs adequate for active distribution networks (ADNs) and microgrids (MGs) with a single grid connection point, have been proposed [2-19]. Modal analysis- and system identification-based methods have been widely used in equivalent modelling. The former approach requires full eigenvalue analysis of linearized state space system model, whereas system identification methods are based on meas ured or simulated system responses and can be further divided into grey-box and black-box equivalents. Unlike grey-box models that require data about the most important physical devices in the analyzed system, black-box models are only focused on matching responses of the detailed and equivalent model without taking into account system structure.

The research on equivalent modelling of DG plants has been devoted to plants consisting of a large number of sametechnology units, with wind farms (WFs) receiving the greatest attention [6]. Equivalent representation of DG plants is commonly derived by aggregating all units in a plant into a single or multi-machine equivalent, which has the same structure as individual units [6-8]. Single machine method is easy to use, but is not able to reflect different dynamic behavior of individual generators [8]. In the case of multimachine method, the main difficulty is a choice of adequate criteria for identifying coherent units that can be represented by the same equivalent generator. Wind speed, real power output and converter control parameters are some of the widely used coherence criteria for WFs and photovoltaic (PV) plants [6-10]. However, coherent generators are typically defined for a single plant operating point. In order to take into account variability in plant operation, probabilistic equivalents of WFs were proposed in [7] and [9].

A grey-box model consisting of an inverter interfaced SG in parallel with a composite load model was proposed in [2, 3] to model ADN. Similarly, a combination of the detailed model of voltage source inverter and a grey-box model of microturbines and fuel cells was suggested as a small-signal MG model in [11]. Black-box DEM in the form of the state space model was proposed in [12]. Voltage and frequency at the 
point of common coupling (PCC) were the model inputs, while real and reactive power at the PCC were used as the outputs. However, model parameters were estimated on the basis of a single ADN response to an external system disturbance, which highly affects model performance. In [13] $\mathrm{ADN}$ real and reactive power responses to small disturbances in voltage at the PCC were simulated by a combination of linear functions and transfer functions (TFs). The main assumption was that the responses corresponding to similar steady state voltage and power at the PCC could be described by the same TF order.

Modelling approaches involving identification of dominant modes in network responses were described in [14-18]. Models in [14, 15] were based on detailed modal analysis, and thus suitable for small signal stability studies only and impractical for large dimensions of today's networks. Prony analysis of measured/simulated network responses was applied in [16] and [17], whereas a combined frequency and time domain approach was proposed in [18]. Unlike the model in [18], models presented in [16] and [17] were valid for small signal analysis only. Equivalents in [17] and [18] were derived and tested for a single network operating point, while correction factors were introduced in [16] to make Prony terms more robust to changes in operating conditions.

Artificial neural networks (ANNs) represent a popular technique for addressing DG equivalent modelling issue. In [4, $11,19]$ ANNs were trained using voltage, current and frequency responses at the PCC. DEMs were suitable for any pre-disturbance operating point as inputs and outputs of ANNs were in the form of normalized deviations from initial steady state. Still, active part of the ADN in [4] and [19] contained only converter connected technologies, whereas the model in [11] was adequate for small disturbances around the analyzed operating point. Tendency to over-fitting and derivation of ANN structure using trial-and-error approach are additional disadvantages. In order to overcome over-fitting problem, a random forest-based model was proposed in [5], but it was only tested for few ADN compositions having the same total power output.

In summary, the major drawbacks of the reported DEMs of DG plants, ADNs and MGs are related to the applicability to a specific technology and to a small number of system operating conditions and disturbances. The disadvantage of the methodologies in terms of plant technology mix is of particular importance as hybrid renewable energy source (HRES) plants have been recognized as a promising solution for obtaining more stable power supply from renewable energy sources (RESs) [20]. HRES plant concept is based on complementary properties of different technologies [20]. They usually consist of non-dispatchable RESs in combination with dispatchable generation and/or storage systems that help mitigate the variability in production of non-dispatchable generators. More controllable power output increases reliability of meeting operator requirements and provides greater opportunity for participating in existing energy and ancillary service markets [20], [21]. So far, the focus of the research has been on optimal HRES plant sizing and operation with respect to economic costs and load following [20]. With an expected increase in the number of large grid-connected HRES plants [22], their influence on system dynamic performance will have to be analyzed as well.

This paper overcomes the identified shortcomings of the past approaches and proposes a methodology for development of DEMs of HRES plants, incorporating different generation and storage technologies, suitable for large system transient stability studies during the year. The approach is based on the analysis of historical plant production data, transmission network (TN) fault statistics and probabilistic modelling of uncertainties associated with system operating condition. First, it enables, in a computationally efficient manner, the identification of the most probable, realistic dynamic behavior of the plant (as seen at the PCC) during the year, or any other predefined time period. Following this, it develops a small set of DEMs in a form of low order TFs to represent HRES plant in transient stability analyses during the whole year. Separate TFs are defined for real and reactive power responses, but they share a common input signal, voltage at the PCC.

Unlike the past methodologies that were focused on modelling dynamic responses of the plant at the PCC with the greatest possible accuracy, the objective of this research was on deriving low order DEMs of the plant that will not compromise the accuracy of the transient stability analysis of the power system as a whole. In other words, priority was given to the overall power system dynamic performance over the actual power response of the considered HRES plant. Focusing on highly accurate modelling of responses of every single element in the power system at any time can result in unnecessary large number of and/or complex network models being used throughout the year, which in turn can cause computationally demanding system stability simulations. Given that the transient stability of a power system is determined by a complex interaction between all elements in the system, HRES plant power responses having different shape in time domain may result in very similar (if not the same) transient system stability performance. Therefore, in this study, HRES plant power responses associated with similar global transient stability behavior were represented by a common DEM regardless of dissimilarities in shape. DEM accuracy was evaluated on the basis of the difference in transient stability results produced by the detailed and equivalent model. Widely used global transient stability indicator, transient stability index (TSI), was used as a criterion to assess the influence of the developed model accuracy on the overall system transient stability and for reduction in number of required models by "merging" similarly performing HRES plant power responses.

The methodology is tested using two HRES plant configurations for a large number of operating scenarios and system disturbances. Both HRES plants are connected to the same IEEE nine-bus test TN model. The studies are performed in MATLAB and DIgSILENT/PowerFactory.

The main contributions of the proposed methodology for equivalent dynamic modelling of HRES plants include: i) Identification of HRES plant operating conditions needed to 
be considered in the modelling process based on historical plant production data (so-called, typical HRES plant compositions), which enables non-computationally demanding modelling procedure; ii) Generation of realistic HRES plant power responses used for DEM development on the basis of typical plant compositions and TN short-circuit fault statistics. In this way, the DEM is capable of reflecting the most probable HRES plant dynamic performance in transient stability studies during a year; iii) Development of DEM from the perspective of the global transient stability results, which provides reduction in the number and/or order of required DEMs compared to common equivalent modelling approaches focusing on highly accurate representation of time domain power responses of the plant; iv) Application of unsupervised data mining methods (clustering techniques) in order to eliminate the need for human decision making; v) Applicability of the methodology to any combination of generation and storage technologies in the HRES plant.

\section{Methodology and Model Development}

The flow chart of the methodology for equivalent modelling of HRES plants for transient stability studies is illustrated in Fig. 1. Inputs and outputs of different stages in the procedure are marked by dashed rectangles. The methodology relies on historical plant production data, statistical data about TN short-circuit fault performance and unsupervised data mining methods (clustering procedures).

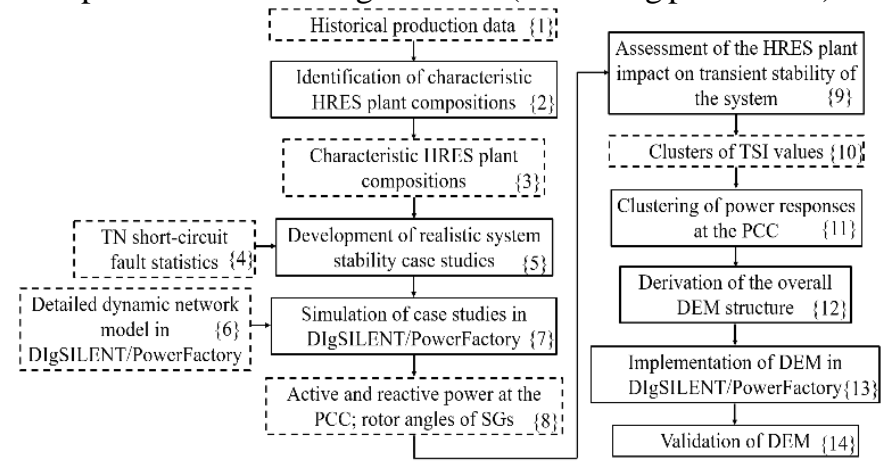

Fig. 1. The flow chart of the equivalent modelling methodology.

Firstly, a set of historical real power outputs of individual plants within the HRES plant is divided into groups according to similarity in technology mixes in the HRES plant, i.e., HRES plant compositions, using an unsupervised clustering technique (block $\{2\}$ in Fig. 1). In this way, characteristic HRES plant compositions (block $\{3\}$ in Fig. 1) during the year, or any other pre-specified time period, are identified. The use of typical instead of all possible plant compositions results in low computational complexity of the equivalent modelling procedure.

In case historical data for a single individual plant in the HRES plant are not available but the real power output of the whole HRES plant was recorded, then the data for this plant can be obtained by subtracting the sum of real power outputs of the remaining individual technologies from the total HRES plant production. However, if production data of the whole HRES plant or more than one individual technology are not available, then the artificial production data have to be generated. Artificial data for PV plants and WFs can be created using solar irradiance and wind speed measurements at the plant location, respectively, and corresponding equations for converting primary energy source into plant power output. On the other hand, the artificial historical production data of dispatchable plants can be obtained as the outputs of the optimal economic dispatch of the HRES plant by minimizing the total plant costs while meeting the pre-specified HRES plant production profile.

Realistic system stability case studies (CSs) used for DEM development are generated in a probabilistic manner (block $\{5\}$ in Fig. 1) and result in the most probable annual HRES plant dynamic performance in transient stability analysis. Typical HRES plant compositions and TN short-circuit fault statistics, as well as uncertainties in production and location of individual plants in the HRES plant, represent a basis for defining CSs. System stability simulations are carried out in DIgSILENT/PowerFactory environment using the detailed HRES plant and TN dynamic model (block $\{7\}$ in Fig. 1). Given that the aim is developing a DEM from the perspective of the overall system stability results, the influence of HRES plant power responses (obtained in the previously defined CSs) on transient stability of the power system is assessed on the basis of the global transient stability indicator - TSI (block $\{9\}$ in Fig. 1). In order to define HRES plant responses resulting in similar transient system stability performance, that is, similar TSI values, groups/clusters of TSI values (block $\{10\}$ in Fig. 1) are identified through an unsupervised clustering process. The number of TSI clusters defines the number of DEMs required for representing the HRES plant in transient stability studies. HRES plant responses resulting in TSI values allocated to the same cluster (block $\{11\}$ in Fig. 1) are represented by a common DEM.

Prior to DEM development (block $\{12\}$ in Fig. 1), power responses are z-normalized as total HRES plant production varies across CSs. Separate parts of DEM are responsible for simulating real and reactive power responses of the HRES plant, but they have the same structure - two blocks connected in series. The first block produces $\mathrm{z}$-normalized power response, which represents an input to the second block that performs inverse z-normalization to obtain power response in absolute units. The block for simulating z-normalized power response is characterized by a time-varying structure - its structure is different for pre-fault, fault and post-fault period. The DEM output is constant before and during the fault (DEM produces different power outputs during these time periods), while a TF is used for simulating power response following the fault clearance. Voltage and real and reactive power at the PCC are TF input and output signals, respectively. A representative system stability CS is defined for each group of HRES plant power responses, and voltage and power responses at the $\mathrm{PCC}$ recorded during that dynamic simulation are used for estimating DEM parameters. Finally, DEM is integrated into the TN model in DIgSILENT/PowerFactory at the PCC (block $\{13\}$ in Fig. 1) and its accuracy is assessed on the basis of the difference between TSI values produced by the detailed and equivalent plant model for the same system 
operating conditions and disturbances (block $\{14\}$ in Fig. 1).

More detailed description of the main steps involved in the modelling procedure is as follows:

\section{A. Identification of Characteristic Annual HRES Plant Compositions}

The patterns in HRES plant production profiles are determined by applying a clustering method to a historical data set of real power outputs of individual generators in the HRES plant. Production levels of individual plants are grouped into a vector at each time step in the considered time period and the vectors are used as inputs to the clustering process. As the historical data set is large, the fuzzy c-means clustering algorithm is used for data clustering. It was selected due to its low computational complexity (i.e., $O(N)$, where $N$ is the number of clustering objects), its property to assign all clustering objects to all clusters with a certain membership degree, which is especially useful in case of clusters not being well separated [23], and its proven efficiency in application in similar clustering tasks in the past [10, 24, 25]. It represents each cluster by its centroid that corresponds to characteristic plant composition in this analysis. As the number of clusters has to be defined in advance, internal clustering evaluation indices, based on assessing inter-cluster and intra-cluster similarity, have been commonly used for estimating the optimal number of clusters [26]. In this study a combination of three widely applied clustering indicators, mean square error (MSE), clustering dispersion index (CDI), and mean index adequacy (MIA) is used [26]. The clustering process is repeated for a range of the number of clusters, the chosen index is calculated for each clustering result and then plotted against the number of clusters. The optimal number of clusters is located at the knee of the curve. If the knee is not noticeable, it is estimated using the method described in [26]. The final number of clusters is determined as the median value of the number of clusters identified by the above three indices.

\section{B. Development of Realistic Case Studies}

A set of realistic CSs is established based on the typical annual HRES plant compositions. Uncertainties in production of individual plants and their location within HRES plant (distance from the PCC) and TN self-clearing short-circuit faults are modelled using probabilistic Monte Carlo (MC) approach. In each MC CS, real power output of individual plant (generation and storage) and the length of lines connecting individual plants to the PCC are sampled uniformly from the pre-specified ranges. As for TN disturbances, in each MC simulation, the location, type and impedance of TN short-circuit fault are selected in a probabilistic manner using TN fault statistical data [27], [28]. Fault location is chosen by sampling a random number $R$ uniformly from range $[0,1]$ and comparing it with the probabilities of a fault occurring at TN buses and lines (see Fig. 2). The probability of fault occurrence at the $i$-th TN element is as follows [27]:

$$
P_{i}=\frac{f_{i}}{\sum_{i=1}^{M} f_{i}}
$$

where $f_{i}$ is an annual fault rate of the $i$-th TN element and $M$ is the number of TN buses and lines. The $i$-th TN element is chosen for fault location if the following is satisfied:

$$
\sum_{j=1}^{i-1} P_{j}<R \leq \sum_{j=1}^{i} P_{j}
$$

In case a line fault is selected, the position of the fault along the line is defined according to the relevant probability distribution function. Fault type is chosen in a similar way as fault location, i.e., by comparing random number with the probabilities of occurrence of four fault types (single-line-toground, double line-to-ground, line-to-line and three-phase fault) at the selected TN element. The selection of the $i$-th fault type is performed according to (1) and (2), but with $P_{i}$ and $f_{i}$ representing the probability of occurrence and annual rate of the $i$-th fault type at the chosen TN element, respectively, and $M$ is equal to 4 (the total number of considered fault types). Finally, fault impedance is sampled from the pre-specified range using the relevant probability distribution.

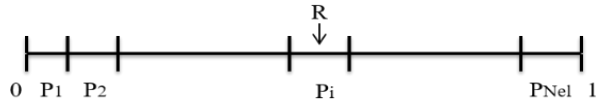

Fig. 2. The illustration of fault location sampling (adapted from [27]).

Transient stability analysis of MC CSs is performed in DIgSILENT/PowerFactory software package using the detailed dynamic model of the HRES plant and TN model. Real and reactive power responses of the HRES plant at the PCC and rotor angles of all SGs in the network are recorded in the simulations.

\section{Assessment of the HRES Plant Impact on Transient Stability of the System}

The HRES plant responses for different CSs are analyzed based on their effect on the overall transient stability of the system. For that purpose, TSI (3) is calculated for each CS [28]:

$$
T S I=\frac{360-\delta_{\max }}{360+\delta_{\max }} \cdot 100 \%,
$$

where $\delta_{\max }$ is the maximum difference between rotor angles of any two SGs in the system at the same time instance. In case $\delta_{\max }$ is larger than 360 degrees, the system is considered to be unstable and the TSI is negative.

The CSs that result in similar TSI values are grouped together and the number of resulting TSI clusters determines the number of DEMs. Grouping is performed using the Kernel Density Estimation (KDE) approach (4) which estimates the probability density function (pdf) of TSI values using the Kernel function [29]:

$$
\hat{f}_{h}(x)=\frac{1}{N h} \sum_{i=1}^{N} K\left(\frac{x-x_{i}}{h}\right),
$$

where $K()$ is nonnegative Kernel function that models the contribution of the individual sample $x_{i}$ to the overall data density and $h$ is the bandwidth (a positive smoothing parameter). Clusters of TSI values are determined based on the local maxima of the estimated pdf [29]. For each clustering object, i.e., calculated TSI value, the nearest local maximum of the pdf is determined through a step-wise hill-climbing procedure and TSI values having the same nearest local maximum are assigned to the same cluster.

The standard Gaussian function with zero mean and standard variance of one is used in this analysis, as it is the 
most common choice for the Kernel function [29]. Estimation of the optimal bandwidth is the most critical step of KDE application, as the bandwidth determines the shape of the estimated pdf. Solve-the-equation plug-in method is chosen for bandwidth estimation as it has demonstrated superior performance, both theoretically and empirically, over a number of other methods [30]. (Note: Unlike the fuzzy cmeans algorithm, which could have been equally used for this purpose, the KDE method does not require the number of clusters to be set in advance, it can identify arbitrary shaped clusters and has strong mathematical background. The drawback of the $\mathrm{KDE}$ is its computational complexity and unavailability in commercial computational packages [31]. This however, was not the case in this study (due to one dimensional data) nor it will be in the case of development of DEM of practical HRES plant.)

\section{Clustering of Power Responses at the PCC}

The clustering of obtained real and reactive power responses based on calculated TSI leads to groups/clusters of responses that need to be represented by a common DEM. Prior to model development though, the power responses have to be normalized as HRES plant output varied from one simulation to the next. Z-normalization (5) is used for that purpose [32]:

$$
\begin{gathered}
z(t)=\frac{Y(t)-Y_{\text {mean }},}{S D(Y(t))}, \\
Y_{\text {mean }}=\frac{1}{n} \sum_{i=1}^{n} Y_{i}, \\
S D(Y(t))=\sqrt{\frac{1}{n-1} \sum_{i=1}^{n}\left(Y_{i}-Y_{\text {mean }}\right)^{2},}
\end{gathered}
$$

where $z(t)$ is the z-standardized power response, $Y(t)$ is power response in absolute units, $Y_{\text {mean }}$ is the average value of the overall power response in absolute units, $S D(Y(t))$ is the standard deviation of the power response in absolute units, $n$ is the number of samples, $Y_{i}$ is instantaneous value of power response in absolute units at the $i$-th time step.

For each cluster of z-normalized power responses a representative response is defined and used for estimating DEM parameters. First, z-normalized real and reactive power responses produced by the same MC CS are grouped into a single vector. Then, a vector characterized by the minimum sum of squared Euclidean distances from the other vectors in the cluster is defined and named a representative vector. Real and reactive part of the representative vector are representative z-normalized real and reactive power response, respectively, whereas the MC case that results in the representative power response is a representative $\mathrm{CS}$ of the cluster.

\section{E. Derivation of the Overall DEM Structure}

Block diagram of the DEM developed for each cluster of power responses is shown in Fig. 3. The real and reactive power responses at the PCC are modelled by separate parts of the DEM though they have the same structure defined by (8):

$$
\begin{array}{r}
z_{E Q, Y}(t)=\left\{\begin{array}{lr}
0, & t<t_{\text {fault }} \\
z_{F a u l t, Y}, & t_{\text {fault }} \leq t \leq t_{\text {clear }}, \\
z_{T F, Y}(t), & t>t_{\text {clear }}
\end{array}\right. \\
Y_{E Q}(t)=Y_{S S}+z_{E Q, Y}(t) \cdot S D_{Y}^{\text {rep }},
\end{array}
$$

where $Z_{E Q, Y}(t)$ is the $\mathrm{z}$-normalized power response of the model, $t_{\text {fault }}$ is the moment of fault occurrence, $t_{\text {clear }}$ is the moment of fault clearing, $Z_{\text {Faul }, Y} Y$ is the value of z-normalized power response of the model during the fault, $z_{T F, Y}(t)$ is the $\mathrm{z}-$ normalized power response of the model after the fault clearance, $Y_{E Q}(t)$ is the power response at the PCC in absolute units (after inverse z-transformation), $Y_{S S}$ is the total power output of the plant before the disturbance, $S D_{Y}{ }^{\text {rep }}$ is the standard deviation of the representative power response in absolute units.

Equation (8a) corresponds to the simulation of $\mathrm{z}$ normalized power response (marked by blue solid rectangles in Fig. 3), whereas (8b) represents the application of inverse normalization to obtain power response in absolute units (marked by blue dashed rectangles in Fig. 3). The DEM part described by ( $8 \mathrm{a})$ is characterized by different structures for pre-fault, fault and post-fault time periods. It can be seen from (8a) that the z-normalized power response before the disturbance is equal to zero as the system is in steady state before the fault occurrence. The z-normalized power response during the fault is assumed to be constant as fault duration is short compared to post-fault recovery period. The average of the representative z-normalized power response during the fault is adopted for modelling the response during this time period (zFault).

The z-normalized power response after the fault clearing is produced by a TF with voltage at the PCC as an input. Different TFs are used for modelling rea and reactive power response. TF parameters are determined through an iterative optimization procedure using voltage and z-normalized power response at the PCC for the representative MC CS as TF input and output, respectively. The order of the TF is gradually increased and its parameters are estimated using the Levenberg-Marquardt algorithm at each step [2, 3]. For each $\mathrm{TF}$ order, TF parameters are estimated by minimizing the sum of squared differences between the representative $\mathrm{z}$ normalized power response and TF output $[2,3]$ :

$$
\min _{\theta} \varepsilon(\theta)=\min _{\theta} \frac{1}{n} \sum_{i=1}^{n}\left(z_{O R G, i}^{r e p}-z_{E Q, i}^{r e p}\right)^{2},
$$

where $\theta$ is a set of TF parameters, $z O R G, i^{\text {rep }}$ is the representative z-normalized power response at the $i$-th time step, and $z_{E Q, i} i^{\text {rep }}$ is the TF output at the $i$-th time step.

Following parameter estimation, the Best Fit Value (BFV) is then calculated for the selected set of parameters using (10) $[2,3]$ :

$$
B F V(\%)=100 \cdot\left(1-\left|\frac{z_{O R G}^{r e p}-z_{E Q}^{r e p}}{z_{O R G}^{r e p}-\bar{z}_{O R G}^{r e p}}\right|\right),
$$

where $\bar{z}_{O R G}^{r e p}$ is the average of the representative z-normalized power response during the post-fault recovery period. The parameter optimization process terminates when the BFV becomes larger than a pre-specified threshold. A threshold of $80 \%$ is adopted in this study $[2,3]$.

Finally, for transformation of z-normalized responses into power response in absolute units the mean value and standard deviation of the actual power response are needed (see (5)). However, this information cannot be known for an arbitrary operating state and short-circuit fault without measuring or 
simulating HRES plant response. Thus, HRES plant power output in steady state for a given forecasted plant composition and the standard deviation of the representative power response in absolute units are used instead of the mean value and standard deviation of the actual power response, respectively.

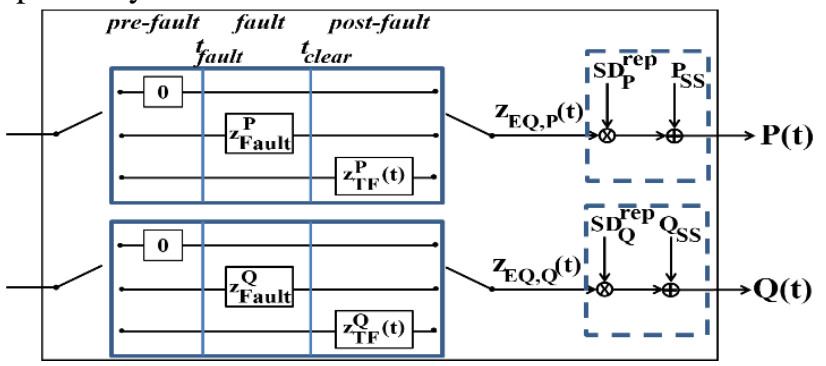

Fig. 3. Block diagram of DEM.

\section{F. Implementation of DEM in DIgSILENT/PowerFactory}

The integration of DEM into DIgSILENT/PowerFactory network model requires an interface in the form of a controllable, constant power load. The load is connected at the PCC and its real and reactive power responses during dynamic simulations are controlled according to (8). The illustration of the DEM concept in DIgSILENT/PowerFactory is presented in Fig. 4.

Built-in DIgSILENT simulation language (DSL) enables development of DEM in the form specified by (8) [33]. Dynamic model developed in DSL environment receives a signal from the network simulation model, voltage at the PCC $\left(u_{P C C}(t)\right.$ in Fig. 4), as input, performs computations defined in (8) and provides pre-specified output signals - reference real and reactive power of the load $\left(P_{E Q}(t)\right.$ and $Q_{E Q}(t)$ in Fig. 4, respectively). Real and reactive power injected at the PCC $\left(P_{P C C}(t)\right.$ and $Q_{P C C}(t)$ in Fig. 4, respectively) correspond to the DEM outputs. The integration time step of the DSL computations is equal to the time step of dynamic network simulations. A set of DEMs, one per cluster of power responses, is created and stored in the software library. DIgSILENT/PowerFactory software provides a feature to automatically select the DEM from the set of available DEMs according to the pre-defined criteria and assigned it to the load model in order to simulate HRES plant dynamic behavior at the PCC in transient stability simulations.

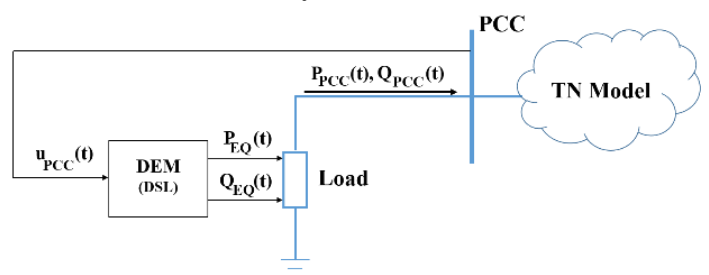

Fig. 4. Illustration of DEM concept in DIgSILENT/PowerFactory.

\section{G. Validation of DEM}

Evaluation of DEM performance is carried out in DIgSILENT/PowerFactory software package for a range of system operating conditions and TN short-circuit faults. Model accuracy is assessed from the point of view of the overall system transient stability, i.e., the error in TSI value:

$$
T S I_{E r r}(\%)=100 \cdot \frac{\left|T S I_{O R G}-T S I_{E Q}\right|}{T S I_{O R G}},
$$

where TSIORG and TSIEQ are TSI values obtained using the detailed model and DEM of the HRES plant, respectively.

\section{TeSt Systems}

The methodology is tested using two CSs involving HRES plant configurations shown in Fig. 5. HRES plants are connected to the same TN represented by the standard IEEE 9bus model [34]. The test systems are modelled in DIgSILENT/PowerFactory software package 2019 [33].

The HRES plant in CS-I consists of three individual plants, namely a WF, a PV plant and a run-of-river hydro power plant (HPP), whereas the HRES plant in CS-II includes, in addition to the above a biomass plant, biogas plant and battery energy storage system (BESS), i.e., six individual plants in total. Installation capacities and dynamic model order of individual plants in both HRES plants are given in Table I. Models of individual plants include the dynamic model of a generation/storage technology and its corresponding control system. All individual plants in CS-I have the same capacity in order to prevent any single energy source dominating the dynamic behavior of the HRES plant, whereas in CS-II the HRES plant configuration corresponds to an optimal technoeconomic solution for the southern part of Greece defined in [35]. The standard fifth-order SG model is used for modelling the hydro generator, while the standard sixth-order model is used for the biomass and biogas SGs [36]. Governors of type IEEEG3, IEEEG1 and GAST are used in the hydro, biomass and biogas generator control system, respectively [36]. The excitation system of all SGs is modelled by IEEE DC1A exciter [36]. The PV plant and the WF are represented in DIgSILENT software by corresponding aggregate models (AMs) obtained by scaling up the model of individual units [33]. Model parameters and power production depend on the number of individual units in service, as it is assumed that units in operation have the same nominal power output. The nominal power of individual units is the same for both types of plants, 2 MW. Further details on PV and WF modelling can be found in [28]. The BESS is represented as a static voltage source equipped with the appropriate control system described in [37].

\section{Results of Simulations}

In both CSs historical production data were scaled so that the maximum production of each individual plant during the analyzed period is equal to its rated power. Historical demand data were scaled so that the maximum load level corresponds to the sum of rated real powers of SGs in the TN and maximum HRES plant production from the historical data set. The scaled historical demand data are assigned to three loads in the TN model (see Fig. 5). The production of SGs in the TN is defined using optimal load flow with the objective of minimizing their total generation cost (generation cost functions are adopted from [34]).

For each typical annual HRES plant composition, 1,000 $\mathrm{MC}$ simulations are carried. In each set of 1,000 simulations, 
the lengths of lines connecting individual plants to the PCC are sampled uniformly between $0.5 \mathrm{~km}$ and $5 \mathrm{~km}$ (in case of larger variation in distance it would be less probable for the individual plants to be connected to the same PCC). The error in generation forecast is modelled by uniformly varying power output of each individual plant in the range of $\pm 5 \%$ around the typical HRES plant composition. Probabilistic fault simulation is carried out using TN fault statistics given in [38] and [39]. It is assumed that all positions along the line have the same probability of being affected by the fault. The fault impedance is sampled uniformly from the range (0-20) $\Omega$ [40]. The plant responses are simulated for $10 \mathrm{~s}$ with the sampling rate of $1 \mathrm{~ms}$ and with a fault occurring at $1 \mathrm{~s}$. The same fault duration of $100 \mathrm{~ms}$ was used in all MC simulations. The simulations are performed in MATLAB and DIgSILENT/PowerFactory (system stability studies) environment.

\section{A. Case Study I}

\section{1) Model development}

The production data used in this CS concern the total production of run-of-river HPPs, PV plants and WFs in Central-Northern Italy for the period 2015-2018. The data with a one-hour sampling rate are available in [41]. As the missing values account for less than $1 \%$ of the dataset, no interpolation was conducted. The MSE, CDI and MIA indices suggest different optimal number of clusters to be used in the fuzzy c-means clustering. They were 9, 6 and 10 respectively, so the median value is chosen and the production data set was divided into 9 clusters. They are presented in Fig. 6 (a) in the form of boxplots. Outliers are marked by red asterisks, whereas whiskers cover $99.3 \%$ of data in the case of normal distribution. Characteristic annual generation compositions are defined by the cluster centroids, e.g., cluster 4 corres ponds to HPP:PV:WF=34\%:30\%:10\% generation mix. Also, 9 clusters of total TN demand corresponding to 9 clusters of plant compositions are defined according to time instance of demand and production data samples (see Fig. 6 (b)).

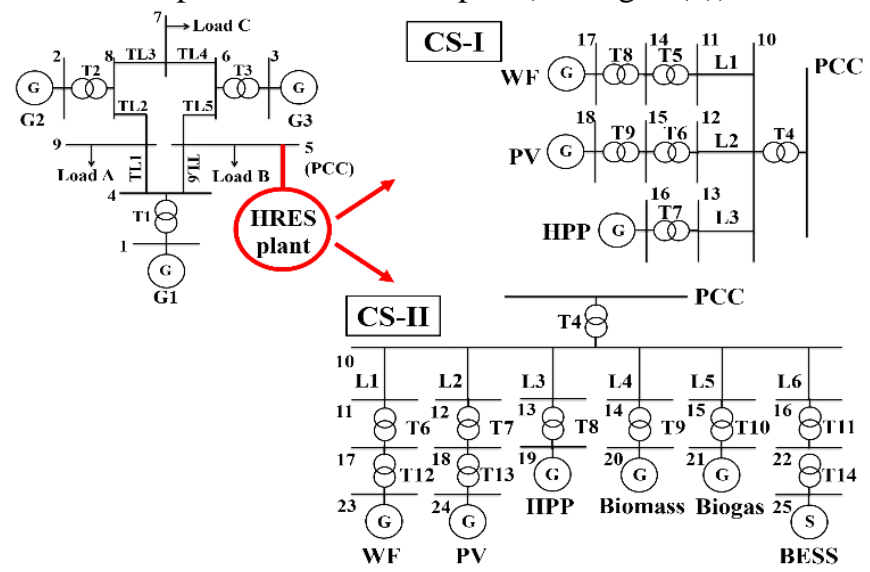

Fig. 5. The schematic diagram of the test HRES plants and IEEE 9-bus network (G: generation technology; S: storage technology).

TABLE I

Installation capacities and model order of the test HRES plants

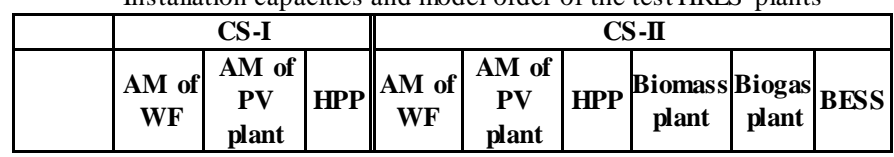

\begin{tabular}{|c|c|c|c||c|c|c|c|c|c|}
\hline $\begin{array}{c}\text { Rated } \\
\text { capacity } \\
\text { (MVA) }\end{array}$ & 210 & 210 & 210 & 170 & 265 & 295 & 76.5 & 76.5 & 125 \\
\hline $\begin{array}{c}\text { Model } \\
\text { order }\end{array}$ & 17 & 14 & 13 & 17 & 14 & 13 & 14 & 11 & 9 \\
\hline $\begin{array}{c}\text { Overall } \\
\text { model } \\
\text { order }\end{array}$ & \multicolumn{3}{|c|}{44} & \multicolumn{5}{|c|}{78} \\
\hline
\end{tabular}

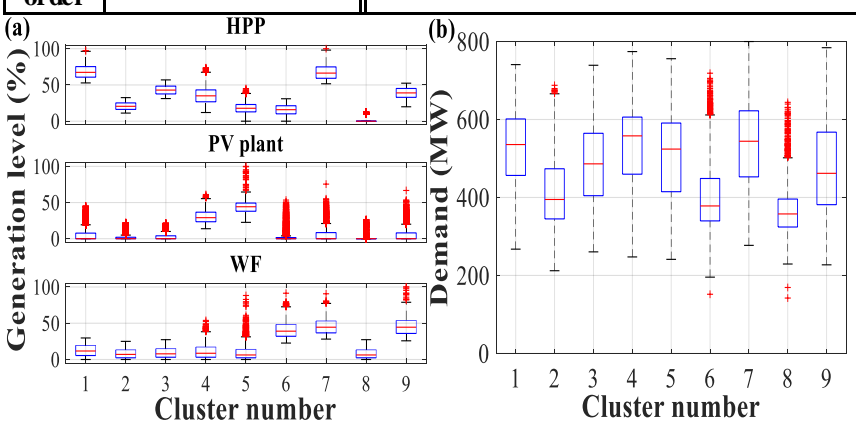

Fig. 6. Typical annual HRES plant compositions (a) and demand levels (b) (base power for production levels is rated power, $210 \mathrm{MVA}$ ).

Fig. 7 (a) shows the pdf of TSI values estimated using the $\mathrm{KDE}$ approach. A great majority of MC simulations is characterized by the TSI being within the range of $80-85$, meaning there is no significant variation in transient stability status regardless of considerable variation in system operating conditions and disturbances. Step-wise hill-climbing method identifies two local maxima corresponding to TSI values of 80.6 and 84.3. This means that two DEMs are required for representing the HRES plant in transient stability studies throughout the year. Two corresponding TSI clusters are shown in Fig. 7 (b) in the form of boxplots. The number of MC cases produced by the same typical plant composition is defined for each TSI cluster and given in Table II. It can be seen that the typical annual HRES plant compositions 6,7 and 9 (the WF loading is about 50\% in those compositions) belong to TSI cluster 2, i.e., they can be represented by DEM 2, whereas the remaining typical generation compositions, 1-5 and 8 , are assigned to DEM 1 . The expected time of use of the corresponding DEMs (defined based on the historical production data set) is shown in Fig. 8 (a). DEM 1 could be confidently used in transient stability simulations pretty much throughout the whole year, except during the first 3 months of the year when both models cover approximately the same number of days and therefore are equally good for modelling the HRES plant in stability studies.
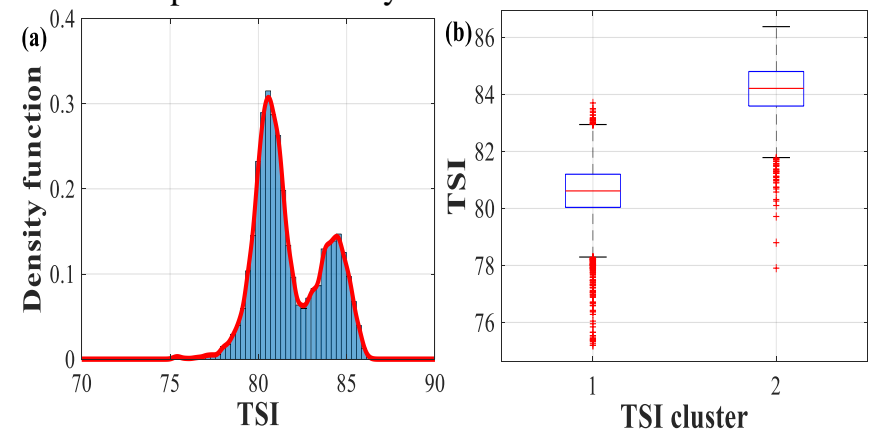

Fig. 7. CS-I: Histogram-pdf of TSI values (a) and TSI clusters (b).

Group of z-normalized real power responses associated with DEM 1 (i.e., TSI cluster 1) is shown in Fig. 8 (b), as an example of clusters of power responses. Representative z- 
normalized power response is shown in black solid line. As can be seen in Fig. 8 (b), responses of considerably different shape are grouped together and assigned to the same DEM. This means that focusing on highly accurate representation of individual plant response is unnecessary from the perspective of obtaining reliable results about the overall system transient stability. Furthermore, the proposed equivalent modelling yields significant simplification (from 44 to 11 states) in HRES plant model for transient stability assessment of the system with embedded HRES plant.

\section{2) Assessment of model accuracy}

The first part of model accuracy assessment is based on MC CSs used for model development. All 9,000 previously defined MC cases are simulated using the equivalent instead of the detailed dynamic model of the HRES plant and the error in TSI value is computed for each MC simulation. The TSIErr indicator given by (11) is shown in Fig. 9 (a) for all analyzed cases. The maximum TSIErr indicator is around $6 \%$ and the median $T S I_{E r r}$ is below $0.5 \%$ for both DEMs, which demonstrates their high accuracy. In order to further test model robustness, the second part of model performance evaluation involves untrained CSs. Namely, the hourly data about the total production of HPPs, PV plants and WFs and total demand in the analyzed region during the year of 2019 are used for generating system operating scenarios. TN shortcircuit faults are defined in the same way as in the case of initial MC simulations. Results of model testing are shown in Fig. 9 (b). Model accuracy is slightly lower than in the case of trained CSs, as there are about $100 \mathrm{MC}$ cases characterized by the $T S I_{E r r}$ indicator being within the range of (10-30)\%. Still, the median error in TSI values is around $1 \%$ for both DEMs.

TABLE II

Number of MC cases perTSI cluster

\begin{tabular}{|l|c|c|c|c|c|c|c|c|c|}
\hline & CC1 & CC2 & CC3 & CC4 & CC5 & CC6 & CC7 & CC8 & CC9 \\
\hline TSI cluster 1 & 890 & 1000 & 1000 & 1000 & 995 & 69 & 14 & 1000 & 37 \\
\hline TSI cluster 2 & 110 & 0 & 0 & 0 & 5 & 931 & 986 & 0 & 963 \\
\hline
\end{tabular}

$\mathrm{CC}=$ characteristic HRES plant composition
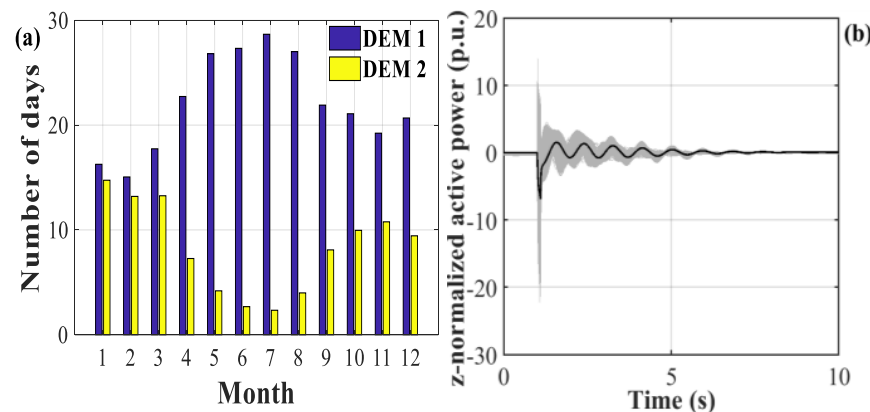

Fig. 8. CS-I: Expected time of use of equivalent models (a) and the first final cluster of z-normalized real power responses (b).
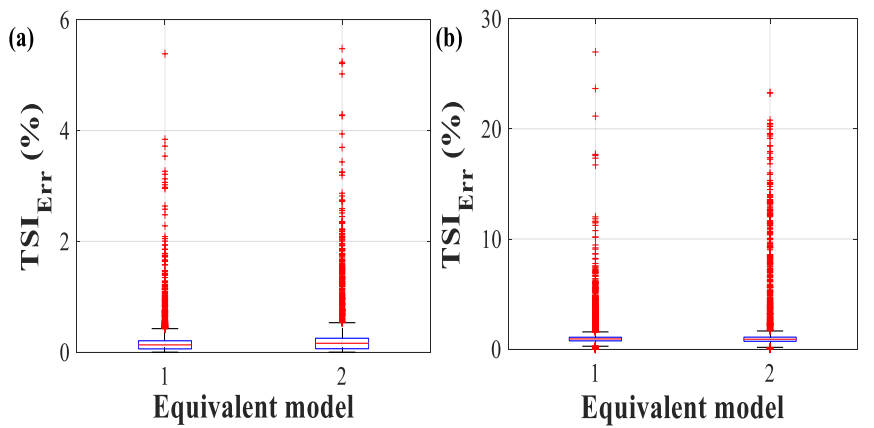

Fig. 9. CS-I: Error in TSI values for trained (a) and untrained (b) CSs.

The accuracy of the DEMs is also compared to the case when the whole HRES plant is replaced by a "negative" constant power load model (NCPLM) as done in many system studies. The NCPLM accuracy is assessed using the MC CSs developed based on the data from the year of 2019 (i.e., cases that were used for DEM validation). The results have shown that the use of the NCPLM doubles the median TSI error: it increases from $0.9 \%$ for the proposed DEMs to $2.5 \%$ for the NCPLM. In addition, $T S I_{E r r}$ indicator is below $4 \%$ for $90 \%$ of the simulated transient stability simulations in the case of the NCPLM, whereas this number is below $1.6 \%$ for the DEMs.

Additionally, though not necessarily required for the purpose that the DEM is developed for, the assessment of DEM accuracy is performed by comparing the time domain responses of the detailed dynamic model of the HRES plant and the responses of the equivalent model. The analysis is carried out for the most dominant model (i.e., the model that can be used most of the time during the year) - DEM 1. The mismatch between the power responses produced by the detailed HRES plant dynamic model and DEM 1 is investigated for MC CSs from TSI cluster 1 (i.e., the training data set used for DEM 1 development) and corresponding cases from the test year (2019) that can be represented by DEM 1. Due to space limitations, only the results for real power responses are illustrated. The period after fault clearing is analyzed as it is relevant for TSI calculation. Fig. 10 provides comparison between z-normalized power responses of DEM 1 and the detailed model for all system conditions allocated to the training DEM 1 data set. Red curves in Fig. 10 represent the envelope of $\mathrm{z}$-normalized power responses produced by DEM 1, which is determined by identifying the minimum and maximum values of all z-normalized DEM 1 responses at each time step of the simulation. The blue line is Z-normalized representative response of the cluster 1 (shown by thick black curve in Fig. 8 (b)). Given that there is small difference between voltage responses at the PCC following a disturbance for the considered system conditions, corresponding z-normalized power responses of DEM 1 are very similar to the z-normalized representative power response (used for estimating DEM 1 parameters). The difference between power responses (in absolute units) produced by the detailed and equivalent model is calculated at each time step of the simulation using the following index:

$$
\Delta Y\left(t_{k}\right)=\frac{Y_{E Q}\left(t_{k}\right)-Y_{O R G}\left(t_{k}\right)}{Y_{O R G}\left(t_{k}\right)} \cdot 100 \%,
$$

where $t_{k}$ is the $k$-th time step of the simulation, $Y_{O R G}\left(t_{k}\right)$ and $Y_{E Q}\left(t_{k}\right)$ is real/reactive power output of the detailed and 
equivalent model, respectively, at the time step $t_{k}$.

Fig. 11 illustrates the values of index (12) calculated for each real power response belonging to the training DEM 1 data set and for each real power response from the year 2019 that can be represented by DEM 1 . The values of the index are shown in the form of boxplots at characteristic time steps of the simulations. In the case of the training data set, the deviation of real power responses of DEM 1 from the original real power responses (i.e., responses produced by the detailed model) is between $-2 \%$ and $2.5 \%$ at each time step for most of the cases. As for the reactive power responses from this data set, DEM 1 accuracy is slightly lower; DEM 1 error at each time step is between $-5.1 \%$ and $0.8 \%$ for most of the responses. However, in most of the cases with DEM 1 error above $\pm 10 \%$, small reactive power output of the HRES plant (below 5 Mvar) is a cause of high values of index given by (12). When it comes to DEM 1 accuracy for the test year (2019), the overall DEM 1 accuracy is similar to the one obtained with the training DEM 1 data set. For most of the responses in this case the DEM 1 error at each time step is within the range $[-1.9,2.3] \%$ and $[-6,2.1] \%$ for real and reactive power responses, respectively. Similar to the training data set, the DEM 1 accuracy for reactive power responses from the test data set is lower compared to the real power responses from the same data set mainly due to small values of the total reactive power output of the HRES plant resulting in high values of index (12).

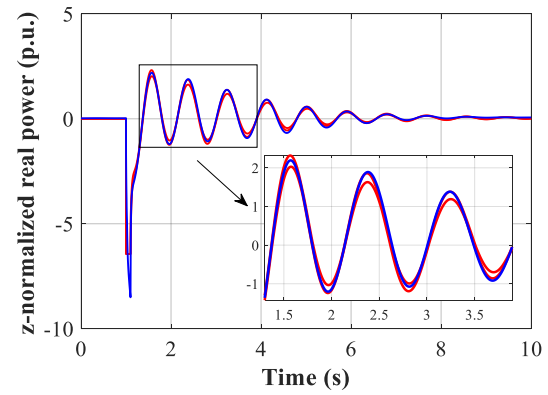

Fig. 10. CS-I: Training DEM 1 data set: Z-normalized representative real power response of the detailed model (blue) and the envelope of $z$-normalized real power responses of DEM 1 (red).
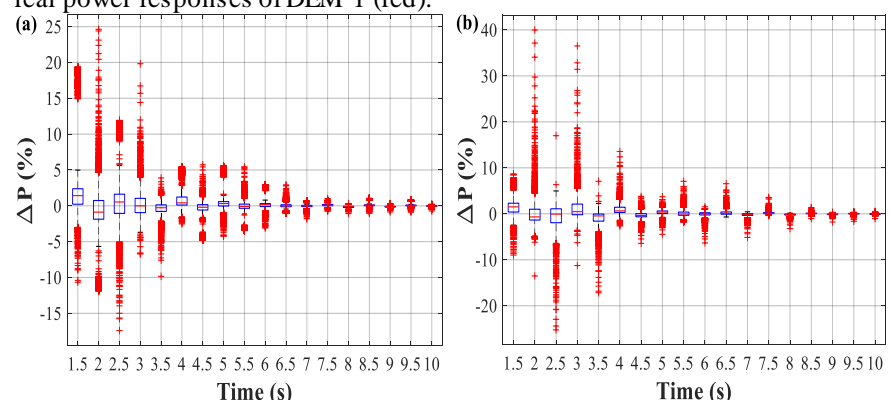
Time (s)

Fig. 11. CS-I: The mismatch between real power responses of the detailed model and DEM 1 ((a): training data set; (b): test data set - year 2019).

\section{3) The influence of data sampling and availability}

In order to assess the influence of the size and sampling rate of historical production data set on DEM accuracy, DEMs of the considered HRES plant have been developed on the basis of three shorter historical periods (six-month summer, six-month winter and one-month period) with one-hour time step as well as for one-month period with a larger six-hour time step. The data from the year of 2017 are used for DEM development as they are characterized by the most dissimilar annual HRES plant production profile compared to the remaining historical years, while the March of 2017 is chosen as one-month period as it is a month with plant compositions having the greatest difference in comparison with the ones used for DEM validation. The accuracy of the new DEMs is evaluated using already defined MC CSs for the year of 2019. Fig. 12 (a) shows the cumulative distribution functions (cdfs) of the TSI error for the DEMs derived on the basis of the reduced historical periods as well as for the original DEMs, while Fig. 12 (b) illustrates the cdfs of the TSI error for DEMs developed based on the one-month data with one-hour and sixhour sampling rates. The $50^{\text {th }}$ and $90^{\text {th }}$ percentile of the cdfs of the TSI error for all DEMs developed based on the three reduced historical data sets with one-hour sampling rate are about $2.3 \%$ and $4 \%$, respectively, which represents a slight reduction in model accuracy compared to the original DEMs derived using the whole four-year historical period $(0.9 \%$ and $1.6 \%$ correspond to the $50^{\text {th }}$ and $90^{\text {th }}$ percentile of the cdf of $T S I_{E r r}$ indicator for the DEMs). As the median TSI error of $2.3 \%$ was obtained for DEMs developed on the basis of only one-month data, this value can be considered as the maximum median TSI error for any DEM of the considered HRES plant developed based on any historical period. When it comes to the impact of data sampling rate on DEM accuracy, very similar, almost identical values of $T S I_{E r r}$ indicator were obtained for DEMs derived based on the one-month period with one-hour and six-hour time step, meaning historical data sampling rate does not have significant impact on the accuracy of TSI value. The reason for a high match between the accuracy of these DEMs is because the increase in sampling rate from one hour to six hours does not affect the ability of the fuzzy c-means clustering algorithm to identify the most probable monthly HRES plant compositions.
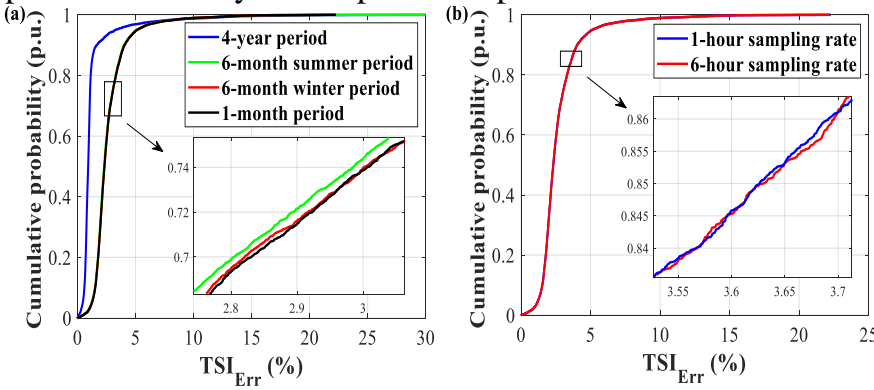

Fig. 12. CS-I: (a): cdfs of TSI error for DEMs developed using 4-year (blue), six-month summer (green), six-month winter (red) and one-month (black) period; (b): cdfs of TSI error for DEMs developed using the one-month data with one-hour (blue) and six-hour (red) sampling rate.

In case new individual plants are integrated into the HRES plant after DEM development, the adequacy of the developed DEMs for representing new HRES plant configuration can be assessed by comparing TSI values produced by these DEMs and the detailed dynamic model of the new HRES plant in probabilistic transient stability simulations. Given that historical data sets required for transient stability analysis for new individual plants cannot be available prior to their commissioning, artificial historical data have to be generated as discussed in Section II A. If, following the transient stability analysis, the use of the existing DEMs results in large 


\section{ACCEPTED VERSION OF THE PAPER}

errors in TSI values (see discussion in Section IV B) then new set of DEMs has to be developed. The previous analysis has shown, however, that both one-month and six-month production data result in the same DEM accuracy. Therefore, the production of individual technologies recorded during the first month of operation of the new HRES plant can be used for the development of the new DEM, and if necessary periodically updated as more data become available.

In order to assess the impact of missing historical production data on DEM performance, two scenarios are analyzed: Scenario I - assuming production data unavailability for a single plant, the WF in this example, and Scenario II assuming production data unavailability for all individual plants in the HRES plant. Given that wind speed and solar irradiance measurements for the considered region were not available, the artificial historical data are generated by varying the available data within the $\pm 20 \%$ range, i.e., by multiplying the available historical data by a random number sampled uniformly from the range $[0.8-1.2]$. The accuracy of the DEMs developed using the artificial historical data is assessed based on the cases used for the validation of the original DEMs. The results of the evaluation of the original DEMs and DEMs developed in Scenario I and II are compared in Fig. 13 using the cdfs of the TSI error. Additionally, the values of the $50^{\text {th }}$ and $90^{\text {th }}$ percentile of TSI error for all three sets of DEMs are given in Table III. It can be seen that the lack of historical data has almost no effect on DEM accuracy. In the case of MC CSs used for the development of the original DEMs, the median $T S I_{E r r}$ is almost the same for all DEMs, while the $90^{\text {th }}$ percentile of TSI error for DEMs from Scenario I and II is higher than the corresponding value for the original DEMs by only $0.04 \%$ and $0.02 \%$, respectively. As for the test 2019 year, the median TSIErr $_{\text {of DEMs from Scenario I and II }(0.88 \% \text { and }}$ $0.84 \%$, respectively) is insignificantly smaller than the median $T S I_{E r r}$ value for the original DEMs $(0.91 \%)$. The reason for this is slightly greater similarity of the data from the test 2019 year with the artificial HRES plant production profiles than with the original historical data.
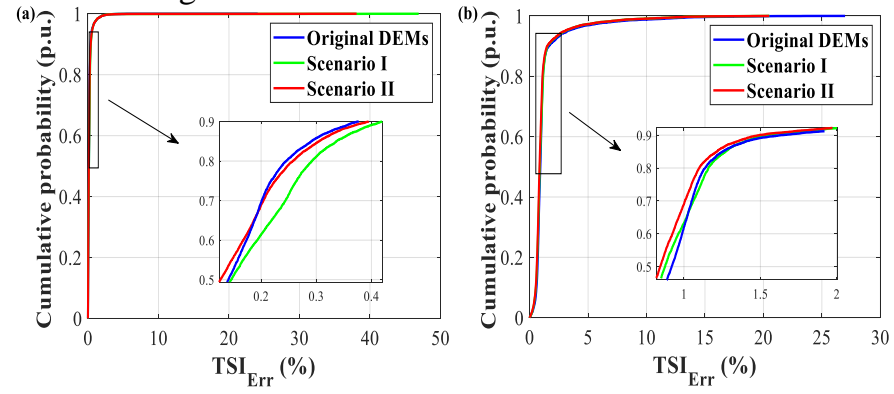

Fig. 13. CS-I: cdfs of $T S I_{E r r}$ indicator for the original DEMs (blue), DEMs from Scenario I (green) and DEMs from Scenario II (red) ((a): MC CSs used for the development of the original DEMs; (b): MC CSs from the test 2019 year).

TABLE III

CS-I: TSI error for three sets of DEMs

\begin{tabular}{|c|c|c|c|c|c|c|}
\hline \multirow{3}{*}{$\boldsymbol{T S I}_{\text {Err }}$} & \multicolumn{2}{|c|}{ MC CSs for the original } & \multicolumn{2}{c|}{ MC CSs from the test 2019 } \\
\cline { 2 - 7 } & $\begin{array}{c}\text { Original development } \\
\text { DEMs }\end{array}$ & $\begin{array}{c}\text { Scenario } \\
\text { I }\end{array}$ & $\begin{array}{c}\text { Scenario } \\
\text { II }\end{array}$ & $\begin{array}{c}\text { Original } \\
\text { DEMs }\end{array}$ & $\begin{array}{c}\text { Scenario } \\
\text { I }\end{array}$ & $\begin{array}{c}\text { Scenario } \\
\text { II }\end{array}$ \\
\hline $\mathbf{5 0}^{\text {th }}$ & $0.14 \%$ & $0.15 \%$ & $0.13 \%$ & $0.91 \%$ & $0.88 \%$ & $0.84 \%$ \\
\hline
\end{tabular}

\begin{tabular}{|c|c|c|c|c|c|c|}
\hline percentile & & & & & & \\
\hline $\begin{array}{c}\mathbf{9 0}^{\text {th }} \\
\text { percentile }\end{array}$ & $0.38 \%$ & $0.42 \%$ & $0.40 \%$ & $1.60 \%$ & $1.54 \%$ & $1.50 \%$ \\
\hline
\end{tabular}

B. Case Study II

\section{1) Model development}

An artificial one-year historical production data set with a one-hour sampling rate was generated based on typical annual solar irradiation, wind speed, electricity price and demand profiles in the analyzed region [35]. At each time step outputs of individual technologies in the HRES plant were determined through an optimization procedure with the aim of maximizing demand provision while minimizing total plant costs. The fuzzy c-means method identified 9 typical annual plant compositions, given in Table IV, yielding 9,000 MC simulations. Fig. 14 (a) shows the pdf of the TSI values estimated using the KDE approach. Similar to CS-I, all MC cases result in transiently stable behavior of the system with small variation in the TSI values. The estimated pdf is characterized by three local maxima, corresponding to the TSI values of 78.3, 81.2 and 92.5, which means that three DEMs are required for modelling annual performance of the considered HRES plant in transient stability studies. Three TSI clusters are presented in Fig. 14 (b).

As in CS-I, majority of the responses resulting from modelling uncertainties associated with fault performance and plant output for a given plant composition get clustered in one TSI cluster. Thus, as in the previous case, the choice of the DEM at any time during the year is predominantly determined by the HRES plant composition. DEM 1 is the most suitable for HRES plant compositions 1, 3 and 8 (compositions with at least one SG in service), while the sixth typical composition, characterized by high production from both the WF and PV plant, is represented by DEM 3. DEM 2 is the most adequate model for the remaining production profiles. Fig. 15 (a) shows the expected time of use of DEMs during the year. DEM 3 has the smallest data coverage and accounts for less than $5 \%$ of the historical data set, whereas DEM 1 and 2 correspond to around $40 \%$ and $55 \%$ of the data set, respectively. DEM 2 is a dominant model in every month, except in winter when DEM 1 and 2 have similar time of use. The model order of DEMs 1 , 2 and 3 are 11,8 , and 9 , respectively, which is considerably smaller than the model order of the detailed HRES plant model (78 state variables). The full mathematical model of DEM 2 is given by (13):

$$
\begin{gathered}
z_{F a u l t, P}(t)=0.4 ; z_{F a u l t, Q}(t)=9.1 \\
z_{T F, P}(t)=\delta^{-1}\left[\frac{-31.4 s^{4}+124.4 s^{3}-5296 s^{2}+1117 s+72.1}{s^{4}+194.9 s^{3}+788.1 s^{2}+1.1 \cdot 10^{4} s+2.1 \cdot 10^{4}}\right] \\
z_{T F, Q}(t)=\mathfrak{\delta}^{-1}\left[\frac{4 s^{4}+1472 s^{3}+3 \cdot 10^{4} s^{2}-1.4 \cdot 10^{4} s-1049}{s^{4}+317 s^{3}+1.3 \cdot 10^{4} s^{2}+6.5 \cdot 10^{4} s+2.1 \cdot 10^{4}}\right] u_{P C C}(t)(13 \mathrm{a}) \\
P_{E Q}(t)=P_{S S}+2.3 \cdot z_{E Q, P}(t) \\
Q_{E Q}(t)=Q_{S S}+6.4 \cdot z_{E Q, Q}(t)
\end{gathered}
$$

where $\mathcal{L}^{-1}$ is inverse Laplace transformation, and $u_{P C C}(t)$ is voltage at the PCC.

TABLE IV

CS-II: Typical annual HRES plant compositions

\begin{tabular}{|c|c|c|c|c|c|c|}
\hline $\begin{array}{c}\text { Cluster } \\
\text { number }\end{array}$ & $\begin{array}{c}\text { WF } \\
\text { (MW) }\end{array}$ & $\begin{array}{c}\text { PV plant } \\
\text { (MW) }\end{array}$ & $\begin{array}{c}\text { HPP } \\
\text { (MW) }\end{array}$ & $\begin{array}{c}\text { Biomass } \\
\text { plant (MW) }\end{array}$ & $\begin{array}{c}\text { Biogas } \\
\text { plant (MW) }\end{array}$ & $\begin{array}{c}\text { BESS } \\
\text { (MW) }\end{array}$ \\
\hline 1 & 12 & 4 & 0 & 64.4 & 58 & 0 \\
\hline
\end{tabular}




\begin{tabular}{|c|c|c|c|c|c|c|}
\hline 2 & 160 & 6 & 0 & 0 & 0 & 0 \\
\hline 3 & 78 & 12 & 0 & 47.9 & 14 & 1.7 \\
\hline 4 & 126 & 80 & 0 & 0 & 0 & -13.6 \\
\hline 5 & 28 & 26 & 0 & 0 & 0 & 91.1 \\
\hline 6 & 148 & 192 & 0 & 0 & 0 & -0.4 \\
\hline 7 & 20 & 132 & 0 & 0 & 0 & -1.5 \\
\hline 8 & 24 & 4 & 128.6 & 0 & 0 & 0.1 \\
\hline 9 & 18 & 202 & 0 & 0 & 0 & -55.4 \\
\hline
\end{tabular}

\section{2) Assessment of model accuracy}

Model accuracy is tested by simulating 9,000 previously defined MC CSs with the DEMs and comparing TSI values obtained in the case of the detailed and equivalent plant model. Fig. 15 (b) illustrates the error in TSI values for all three DEMs in the form of boxplots. The maximum error in TSI values is below $20 \%$ for all MC simulations. Furthermore,

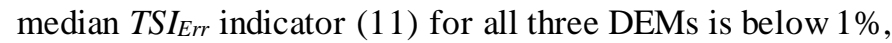
confirming that the equivalent modelling approach provides DEMs of high accuracy.

As in CS-I, the suitability of the NCPLM for representing the whole HRES plant is analyzed based on the MC CSs used for DEM evaluation. The use of the NCPLM instead of the proposed DEMs results in larger TSI error in this CS as well. For the NCPLM, the $50^{\text {th }}$ and $90^{\text {th }}$ percentile of the cdf of $T S I_{E r r}$ indicator are $4 \%$ and $5.6 \%$, respectively. On the other hand, the developed DEMs are characterized by around $1 \%$ and $2.9 \%$ error for the $50^{\text {th }}$ and $90^{\text {th }}$ percentiles, respectively.
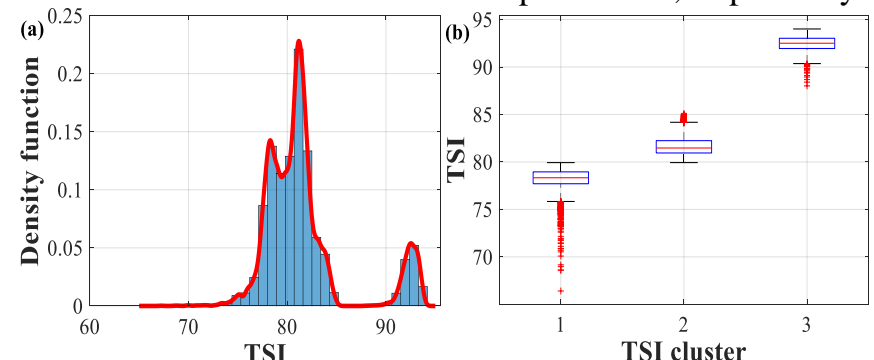

Fig. 14. CS-II: Histogram-pdf of TSI values (a) and TSI clusters (b).
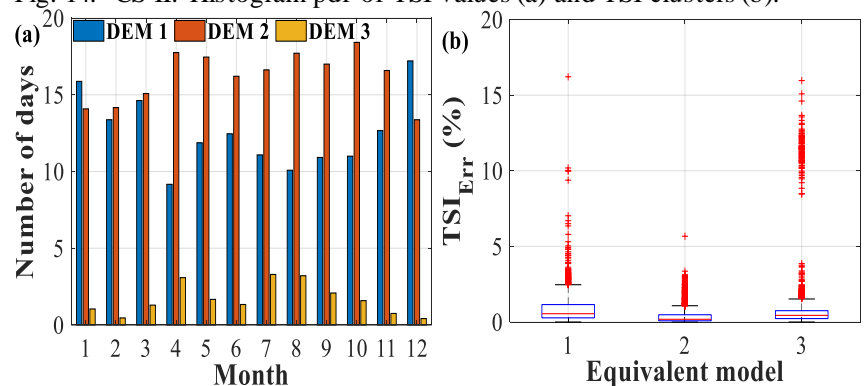

Fig. 15. CS-II: The expected time of use of equivalent models (a) and model accuracy (b).

(Note: Theoretically, the TSI values can be in the range from - 100 to 100 . The system is more stable if the TSI value is closer to 100, while the system stability boundary corresponds to zero TSI value. In both CSs presented in the paper, the simulated TSI values were far away from the transient stability limit (TSI value was in the range 70-95). Thus, even a considerable TSI error of 50\%, calculated by (11), would not result in an inaccurate assessment of the transient system stability status. The actual errors in TSI value obtained when comparing performance of the detail model and DEMs were within a few percent, which implies that the transient stability status of the system with DEMs remains practically the same as with the full-scale HRES plant model. Even if the original TSI values were much smaller, say about 5 , the $50 \%$ error would give a range between 2.5 and 7.5 which still corresponds to the transient behavior and status of the system with the detailed model. Based on the above, one could safely set a (very) conservative limit on the TSI error variation of $\pm 20 \%$ which would guarantee practically the same transient system behavior.)

\section{CONCLUSION}

This paper presented the methodology for dynamic equivalent modelling of HRES plants for transient stability studies. The methodology is applicable to any technology mix in plants with a single point of connection to TN. The proposed equivalent modelling approach is based on historical plant production and $\mathrm{TN}$ fault statistics and results in a dynamic model in the form of a TF with the voltage and real and reactive power at the PCC as the input and outputs, respectively. Procedures for generating the artificial production data sets in case of historical data unavailability are given as well. Still, it should be noted that it is highly unlikely that there would be absolutely no information about real power output of any individual technology in the HRES plant as one would assume that the plant was installed after conducting detailed analysis on power potential of RESs at the considered location.

The focus of the study was on development of DEMs of HRES plant suitable for the sufficiently accurate assessment of the overall system transient stability as described by TSI value. This was judged to be more important and more useful for practical large system studies than development of highly accurate dynamic models of HRES plant as seen from the PCC.

The methodology was tested on two HRES plant configurations connected to the same TN. In both CSs, a few low-order DEMs were required for representing the plant in system transient stability analyses throughout the year. Model performance was tested for a wide range of operating conditions and TN short-circuit faults. The median error in transient stability assessment was below $1 \%$ for all DEMs of the test HRES plants. Considering high accuracy of transient stability assessment and low order of the equivalent models, the proposed methodology can clearly provide computationally efficient and accurate large network transient stability analysis with embedded HRES plants. The proposed approach is highly suitable for practical applications as the information about the past HRES plant production profile is the only prerequisite for selecting the adequate DEM for transient stability studies at any time during the year. Furthermore, in the case of system dynamic security assessment the forecasted plant output and composition can be used to select the most appropriate DEM from the library of previously developed models. The number of models in the library and time granularity of the models (e.g., the time period of the year covered by a particular model) is user dependent as these models can be developed offline and stored 
in the library for future use.

\section{REFERENCES}

[1] T. Ackermann, G. Andersson, and L. Söder, "Distributed generation: a definition," Electr. Pow. Syst. Res., vol. 57, no. 3, pp. 195-204, Apr. 2001.

[2] S. M. Zali, J. V. Milanović, "Generic model of active distribution network for large power system stability studies," IEEE Trans. Power Syst., vol. 28, no. 3, pp. 3126-3133, Aug. 2013.

[3] J. V. Milanović, S. M. Zali, "Validation of equivalent dynamic model of active distribution network cell," IEEE Trans. Power. Syst., vol. 28, no. 3, pp. 2101-2110, Aug. 2013.

[4] J. Denecke, I. Erlich, "Dynamic equivalents of active distribution networks," in Proc. IEEE Power Energy Soc. Gen. Meeting, 2017, pp. 15.

[5] X. Wu, "New approaches to dynamic equivalent of active distribution network for transient analysis," Ph.D. dissertation, RWTH Aachen Univ., Aachen, Germany, 2016.

[6] P. Li, W. Gu, L. Wang, B. Xu, M. Wu, and W. Shen, "Dynamic equivalent modeling of two-staged photovoltaic power station clusters based on dynamic affinity propagation clustering algorithm," Int. J. Elec. Power., vol. 95, pp. 463-475, Feb. 2018.

[7] M. Ali, I. S. Ilie, J. V. Milanović, G. Chicco, "Wind farm model aggregation using probabilistic clustering," IEEE Trans. Power Syst., vol. 28, no. 1, pp. 309-316, Feb. 2013.

[8] J. Zou, C. Peng, Y. Yan, H. Zheng, and Y. Li, "A survey of dynamic equivalent modeling for wind farm," Renew. Sustain. Energy Rev., vol. 40, pp. 956-963, Dec. 2014.

[9] H. Zhou, P. Ju, Y. Xue, and J. Zhu, "Probabilistic equivalent model of DFIG-based wind farms and its application in stability analysis," J. Mod. Power Syst. Clean Energy, vol. 4, no. 2, pp. 248-255, Apr. 2016.

[10] J. Zou, C. Peng, H. Xu, and Y. Yan, "A fuzzy clustering algorithm-based dynamic equivalent modeling method for wind farm with DFIG," IEEE Trans. Energy Convers., vol. 30, no. 4, pp. 1329-1337, Dec. 2015.

[11] F. de Oliveira Resende, "Contributions for microgrids dynamic modelling and operation," Ph.D. dissertation, Eng. Faculty, Porto Univ., Porto, Portugal, 2007.

[12] X. S. Feng, "Dynamic equivalencing of distribution network with embedded generation," Ph.D. dissertation, Univ. of Edinburgh, Edinburgh, U.K., 2012.

[13] E. O. Kontis, T. A. Papadopoulos, M. H. Syed, E. Guillo-Sansano, G. M Burt, and G. K. Papagiannis, "Artificial-intelligence method for the derivation of generic aggregated dynamic equivalent models," IEEE Trans. Power Syst., vol. 34, no. 4, pp. 2947 - 2956, July 2019.

[14] A. Ishchenko, J. M. A. Myrzik, and W. L. Kling, "Dynamic equivalencing of distribution networks with dispersed generation using Hankel norm approximation," IET Gen. Transm. Distrib., vol. 1, no. 5, Sept. 2007.

[15] M. Rasheduzzaman, J. A. Mueller, and J. W. Kimball, "Reduced-order small-signal model of microgrid systems," IEEE Trans. Sustain. Energy, vol. 6, no. 4, pp. 1292-1305, Oct. 2015.

[16] P. N. Papadopoulos, G. K. Papagiannis, A. J. Roscoe, P. Crolla, T. A. Papadopoulos, and G. M. Burt, "Measurement-based analysis of the dynamic performance of microgrids using system identification techniques," IET Gen. Transm. Distrib., vol. 9, no. 1, pp. 90-103, Jan. 2015.

[17] S. M. Zali, J. V. Milanović, "Dynamic equivalent model of distribution network cell using Prony analysis and nonlinear least square optimization," in Proc. IEEE Bucharest PowerTech, 2009, pp. 1-6.

[18] T. A. Papadopoulos, A. I. Chrysochos, E. O. Kontis, P. N Papadopoulos, and G. K. Papagiannis, "Measurement-based hybrid approach for ringdown analysis of power systems," IEEE Trans. Power Syst., vol. 31, no. 6, pp. 4435-4446, Nov. 2016.

[19] A. M. Azmy, I. Erlich, and P. Sowa, "Artificial neural network-based dynamic equivalents for distribution systems containing active sources," Proc. Inst. Elect. Eng. Gen. Transm. Distrib., vol. 151, no. 6, pp. 681688, Nov. 2004.

[20] A. Hina Fathima, K. Palanisamy, "Optimization in microgrids with hybrid energy systems - a review," Renew. Sustain. Energy Rev., vol. 45, pp. 431-446, May 2015.

[21] J. Chen, H. E. Garcia, "Economic optimization of operations of hybrid energy systems under variable energy markets," Appl. Energy, vol. 177, pp. 11-24, Sept. 2016.
[22] W. Gorman, A. Mills, M. Bolinger, R. Wiser, N. G. Singhal, E. Ela, E. O'Shaughnessy, "Motivations and options for deploying hybrid generator-plus-battery projects within the bulk power system," Electr. J., vol. 33, no. 5, pp. 1-15, June 2020.

[23] R. Xu and D. Wunsch, "Survey of clustering algorithms," IEEE Trans. Neural Netw., vol. 16, no. 3, pp. 645-678, May 2005.

[24] M. Begovic, A. Pregelj, A.Rohatgi, D. Novosel, "Impact of Renewable Distributed Generation on Power Systems," in Proc. 34th Hawaii Int. Conf. Syst. Sci., 2001, pp. 654-663.

[25] T. C. Havens, J .C. Bezdek, C. Leckie, L. O. Hall, M. Palaniswami, "Fuzzy c-means algorithms for very large data," IEEE Trans. Fuzzy Syst., vol. 20, no. 6, pp. 1130-1146, Dec. 2012.

[26] G. J. Tsekouras, P. B. Kotoulas, C. D. Tsirekis, E. N. Dialynas, and N. D. Hatziargyriou, "A pattern recognition methodology for evaluation of load profiles and typical days of large electricity customers," Electr. Pow. Syst. Res., vol. 78, no. 9, pp. 1494-1510, Sept. 2008.

[27] W. Li, Risk Assessment of Power Systems: Models, Methods, and Applications, 2nd ed. Hoboken, New Jersey: John Wiley \& Sons, Inc., 2014.

[28] P. N. Papadopoulos and J. V. Milanović, "Probabilistic framework for transient stability assessment of power systems with high penetration of renewable generation," IEEE Trans. Power Syst., vol. 32, no. 4, pp. 3078-3088, July 2017.

[29] J. Han, M. Kamber, J. Pei, Data Mining Concepts and Techniques, 3rd ed. Waltam, USA: Elsevier Inc., 2012.

[30] M. C. Jones, J. S. Marron, S. J. Sheather, "A brief survey of bandwidth selection for density estimation," J. Amer. Statist. Assoc., vol. 91, no. 433, pp. 401-407, Mar. 1996.

[31] V. C. Raykar, R. Duraiswami, "Fast optimal bandwidth selection for kernel density estimation," in Proc. 6th SIAM Int. Conf. Data Mining, 2006, pp. 524-528.

[32] I. B. Mohamad, D. Usman, "Standardization and its effects on k-means clustering algorithm," Res. J. Appl. Sci. Eng. Technol., vol. 6, no. 17, pp. 3299-3303, Sept. 2013.

[33] DIgSILENT PowerFactory 2019 User Manual. DIgSILENT GmbH, 2019.

[34] R. D. Zimmerman, C. E. Murillo-Sanchez, R. J. Thomas, "MATPOWER: Steady-state operations, planning, and analysis tools for power systems research and education," IEEE Trans. Power Syst., vol. 26, no. 1, pp. 12-19, Feb. 2011.

[35] "D5.2 - Assessment of the combination of RES and storage alternatives under the Manager Energy for Hybrid Plants (ME4HP) and REDOX flow battery characterization," CROSSBOW Project, 2019, [Online]. Available: http://crossbowproject.eu/deliverables/.

[36] P. Kundur, Power system stability and control. New York, NY, USA: McGraw-Hill, 1994.

[37] A. Adrees, J. V. Milanović, "Study of frequency response in power system with renewable generation and energy storage," in Proc. Power Syst. Comput. Conf., 2016, pp. 1-7.

[38] Y. Zhang, "Techno-economic Assessment of Voltage Sag Performance and Mitigation," Ph.D. dissertation, Sch. Electr. Electron. Eng., Univ. of Manchester, Manchester, U.K., 2008.

[39] R. C. Leborgne, G. Olguin, J. M. Carvalho Filho, M. H. J. Bollen, "Differences in voltage dip exposure depending upon phase-to-phase and phase-to-neutral monitoring connections," IEEE Trans. Power Del., vol. 22, no. 2, pp. 1153-1159, Apr. 2007.

[40] K. C. Cebrian, L. Camilo, N. Kagan, N. M. Matsuo, H. Arango, "Consideration of voltage sags disruption risks in distribution planning studies," in Proc. CIRED 18th Int. Conf. Electr. Distrib., 2005, pp. 1-4.

[41] ENTSO-E Transparency Platform. [Online] Available: https://transparency.entsoe.eu.

Ana Radovanović (S'18) received the B.Sc. and M.Sc. degrees in electrical enginering and computer science from the University of Belgrade, Belgrade, Serbia, in 2013 and 2014, respectively. She is currently pursuing the Ph.D. degree at the Department of Electrical and Electronic Engineering, the University of Manchester, Manchester, U.K., in the areas of renewable energy sources and power systemdynamics.

Jovica V. Milanović (M'95-SM'98-F'10) received Dipl.Ing. and M.Sc degrees from the University of Belgrade, Belgrade, Yugoslavia, a Ph.D. degree from the University of Newcastle, Newcastle, Australia, and a D.Sc. degree from the University of Manchester, Manchester, U.K., all in Electrical 


\section{ACCEPTED VERSION OF THE PAPER}

Engineering. He is currently a Professor of Electrical Power Engineering and a Deputy Head of Department of Electrical and Electronic Engineering at the University of Manchester, Manchester, U.K., and a Visiting Professor at the University of Novi Sad, Novi Sad, Serbia and University of Belgrade, Belgrade, Serbia. Professor Milanović is a Chartered Engineer in the UK,

Foreign member of the Serbian Academy of Engineering Sciences, Fellow of the IET, Fellow of the IEEE, Distinguished IEEE PES Lecturer and Chair of the IEEE Herman Halperin Transmission and Distribution Award Committee. He was a member of the IEEE PES Governing Board, for six years, as Regional Representative for Europe, Middle East and Africa and a member and vice-chair of PES Fellows Evaluation Committee. 